\title{
Bioethical looked in patients with mesentéric thrombosis in terminal stadium
}

\begin{abstract}
Is carried out a clinical exhibition and valuation bioética in patients that suffer of thrombosis mesentérica in terminal stadium, where the members of the medical team should be prepared to offer support in this dramatic situation, to alleviate their suffering and the fears that they suffer, also the emotional support to the relatives, their physical integrity and their individual rights, as well as to analyze the informed knowledge of their fatal outcome.
\end{abstract}

Keywords: bioethical, thrombosis mesentérica, palliative sedation
Volume 3 Issue I - 2019

\author{
Enrique Arce Morera \\ Specialist of II degree in Physical Medicine and Rehabilitation, \\ Master in Natural Medicine and Bioenergética in A.P.S. Assistant, \\ Educational clínic Dr. “Tomás Romay”, Cuba \\ Correspondence: Enrique Arce Morera, Specialist of II degree \\ in Physical Medicine and Rehabilitation, Master in Natural \\ Medicine and Bioenergética in A.P.S. Assistant. Educational clínic \\ Dr."Tomás Romay", Artemisa, Cuba, \\ Email earce@infomed.sld.cu
}

Received: December 18, 2018 | Published: January 24, 2019

\section{Introduction}

The enteritis isquémica or isquemia mesentérica, also denominated as thrombosis mesentérica are due to the faulty circulation of the thin intestine (from a simple isquemia until the total necrosis of the intestine). Its can have different presentations (isquémicas, trombóticas or embólicas). When being occluded the artery superior mesenterica in a sharp way, the sanguine watering is interrupted to the yeyuno-ileon and the right colon causing a massive intestinal infarto.

It is more frequently in the masculine sex, in proportion of three at two and between 55 and 60 years or more. ${ }^{2}$

Clinical square: Sudden and intense abdominal pain, it can be colic type accompanied by bloody depositions, vomits and abdominal distension that doctors makes think of an intestinal obstruction of mechanical cause, disappearance of the abdominal noises, more late by the imbalance hidroelectrolitico acid-base that takes the gangrened intestine it can be perforated until arriving to a peritonitis.

Diagnosis: This intestinal lesion is not frequent and it becomes complex to diagnose, because it confuses with: a sharp colecistitis, sharp pancreatitis, perforated ulcer and intestinal obstruction. The complementary studies as the abdominal ultrasound, ecografia with veined Doppler, tomografias and arteriografias is of utility to arrive to the same one.

Treatment: A previous treatment of medical character exists with anticoagulants and vasodilatadores, to pass to the surgical component by means of laparotomía (exploration of the abdominal cavity) with observation of having perspired, the brown purple or red coloration of the intestinal handle and absence of beats in the trunk of the superior mesenteric artery. If local necrosis exists doctor proceed with the resection of the segment ( $50 \%$ of the cases), relaparotomia (second look). If the isquemia is reversible they make at revascularization or embolóctomia according to the case. If the isquemia persists and all the intestinal handles have to be extirpated, there is not possibility of survival and in consequence the death in is ours.

This approach of vital, given incompatibility the limitation of the therapeutic effort is notified the relatives the fatal outcome, without the possibility of another treatment alternative and doctors decides the constant sedation, support for via parenteral and control of vital signs, days later, after long agony and its relatives' suffering, in these patients it happens a process of auto destruction and in anasarca state, they die.

In the following article a valuation bioética is exposed in the patients that suffered or they will suffer of this terrible illness.

Development: The medical bioética is capable to offer an ethical foundation to the growing necessities of the services of health, to prosecute and to establish rules in the communities of professionals. The development of the bioethical, it has put in discussion topics of vital importance in relation to the rights and the patients' duties. It is in fact the limitation of the therapeutic effort one of the most polemic to world level, because they are in game aspects of first order like they are the patient's life, the family dilemma and the taking of decisions for the specialist doctors, this clinical complexity justifies to think about contrarily if the most appropriate concept would be sedation terminal. $^{3}$

In the peculiar case of the patients with thrombosis mesenteric, with the total extirpation of their bowels after several surgical interventions and without possibility of survival, does think about a quite complex ethical dilemma for the relative little time of survival in these cases that differ of the patients in the cancerous processes as the history of the illness in the terminal stadium of Sigmund Freud: ${ }^{4}$ Euthanasia or appropriation?, where he thought about moral problems, to happen before the birth of the bioética and to be classified like an euthanasia active insinuation according to the rule of twice as much effect.

According to the author of the work: Euthanasia: a reflection from the Bioethical ${ }^{5}$ look are of the approach that the professional and scientific answer to the patient's necessities in terminal situation, is in the palliative cares that according to a definition of the World Organization of the Health (OMS), they seek to be in charge of the patients whose illnesses don't respond to healing treatments. It is in these fundamental cases the control of the pain, of other symptoms and of the psychological, social and spiritual problems. The objective is to reach the best quality of life for the sick person and it stops its family suffer, to get these sick persons so they live with fullness its last months or days.

In the last years one has interest for the knowledge, valuation and the patients' appropriate treatment in terminal situation; they are 
numerous the publications that approach the topic. It is the medicine palliative that is in charge of the global attention and support of the patients during. ${ }^{6,7}$

The limitation of the therapeutic effort is a good clinical practice that differs from the euthanasia, the attended suicide and the right to die that are not alternative acceptable in the medicine; to be contrary to the deontología and to the medical ethics, therefore they are not neither moral neither legally acceptable in the Cuban society. The doctor should have special sensibility and moral and human integrity to face the decisions at the end of the life of his patients. ${ }^{8}$

The palliative sedation is a therapeutic procedure dedicated to the relief of the refractory symptoms that can appear in the context from the sick person to the end of the life. The following requirements should be completed: refractory symptom, terminal illness, consent and as much as possible to be endorsed by a second medical opinion. The palliative sedation is not euthanasia and the differences are in the objective, the process and the result. ${ }^{9}$

The sedation effect for the rebellious pain in the survival of patient in terminal stadium is a very debated topic, the percentage of appropriate sedations and the general process hardly improved with the setting-up of a protocol for this end, for what is necessary revaluar its effectiveness.

Studies directed among the years 1990 and 2009 don't show that the death of patients that suffer the sedation for the rebellious pain accelerates. Some authors conclude that sedation doesn't accelerate the death and it demands that the principle of double effect is unnecessary in this debate. ${ }^{3,10}$

Caution is required in the interpretation of results of the studies by absence of evidence of the effects of the sedation in the survival. Far from limiting the doctor's action, the principle of double effect that demands us to allow us to make the distinction among the palliative sedation for the rebellious pain and eutanasai. ${ }^{11}$

\section{Conclusion}

The decision of limiting some procedure of vital support doesn't mean that the therapeutic effort concludes. In this context, the palliative cares pass to a first line assistance, where the sedation, the analgesia and the psychological support, they are indispensable supports and of great responsibility. The limitation of the beginning of certain measures of vital support refers to the taking of decisions of not using certain procedures or therapeutic specific in a patient that hopelessly will die. Of course that is not of the deliberate omission of a due and necessary care for the sick person survival, but of not beginning a treatment when it lacks sense according to the modernized medical approaches. In occasions the incorporation of new measures of vital support is limited, but they already stay those established.

Without a doubt, the Institutions of Collective Medical Attendance, the members of the team of health and of course the families should involve in the palliative cares in these patients of terminal stadium, because, although we don't want, we see in the suffering and the death of the other one, our own death. Each human being is only and irrepetible and it deserves respect along all his life, from the conception until the death. ${ }^{12}$

The importance of the informed consent to the patient of its dramatic situation should be necessary in these cases of imminent death for thrombosis mesentérica, because the determination is usually taken by the medical team and official statement to the relatives.

Then, serious ethical that the patient ignored their final outcome before they are applied the procedures of terminal sedation?

\section{Acknowledgments}

None.

\section{Conflicts of interest}

The authors declare there is no conflicts of interest.

\section{References}

1. Soler VR. Surgery of the abdomen. Editorial Medical Sciences. Havana. 2010;13:137.

2. Robbins S. Treated L. of pathology. Revolutionary edition: Havana; 2nd ed. 1973:669.

3. Santos D, Valle AD, Barlocco B. et al. Palliative sedation: experience in an unit of cares palliatives of Montevideo. Rev Méd Urug. 2009;25(2).

4. Figueroa G. Bioética of the death of Sigmund Freud. Euthanasia or appropriation? Rev méd Chile. 2011;139(4):529-534.

5. https://revistas.unimilitar.edu.co/index.php/rlbi/article/view/1141

6. Espinosa RA. Palliatives cares to sick persons in terminal phase adults in the primary attention of health. Doctórate thesis. Cuba: Facultad Medical Sciences of Cienfuegos; 2010.

7. Betancourt BJ. Limitation of the therapeutic effort versus euthanasia: a bioétic reflection. Rev Hum Med. 2011;11(2).

8. Ojeda MM, Gómez SM, Grau AJ, et al. The evaluation of the impact of programs of Palliatives cares: a study of the quality of life in patient of an university hospital. University of Veracruz. 2009;19(1).

9. Serrano JR. About the injustice of the euthanasia. The use of the compassion like moral mask. It indexes to new documents european bioétics. University of the Savanna. 2006.

10. Benoît F Leheup, Elise Piot, Xavier Ducrocq, et al. Principle of double effect and sedation for intractable distress: Reflexion on survival in sedated patients. La Presse Médicale. 2012;41(10):927-932.

11. Mateos-Nozal J, García CL, Montero EB, et al. Analysis of the palliative sedation in hospitalized bigger patients: effectiveness of a protocol. Rev Esp Geriatr Gerontol. 2016;51(3):132-139.

12. http://www.um.edu.uy/docs/revista_agosto_rcb.pdf\#page=17 